\title{
Расчет эффективности удвоения частоты излучения субтерагерцового гиротрона за счет решеточной нелинейности в монокристаллической пластине $\ln P$
}

\author{
() В.В. Румянцев ${ }^{1}$, А.П. Фокин ${ }^{2}$, А.А. Дубинов ${ }^{1}$, С.С. Морозов ${ }^{3}$, А.А. Богдашов ${ }^{2}$, В.В. Паршин ${ }^{2}$, \\ М.Ю. Глявин ${ }^{2}$, В.И. Гавриленко ${ }^{1}$, С.В. Морозов ${ }^{1, \uparrow}$ \\ ${ }^{1}$ Институт фризики микроструктур Российской академии наук, \\ 603950 Нижний Новгород, Россия \\ ${ }^{2}$ Институт прикладной физики Российской академии наук, \\ 603950 Нижний Новгород, Россия \\ ${ }^{3}$ Нижегородский государственный университет им. Лобачевского, \\ 603950 Нижний Новгород, Россия \\ ฯ E-mail: more@ipm.sci-nnov.ru
}

Поступила в Редакцию 12 апреля 2021 г.

В окончательной редакции 19 апреля 2021 г.

Принята к публикации 19 апреля 2021 г.

\begin{abstract}
Выполнен расчет эффективности генерации второй гармоники для субтерагерцового гиротронного излучения в пластине монокристаллического InP, встраиваемой в конический металлический волновод. Для различных величин потерь на удвоенной частоте (зависящих от степени чистоты кристалла InP) выявлены оптимальные размеры пластины, обеспечивающие наиболее эффективное преобразование частоты. Так, коэффициент преобразования $f \rightarrow 2 f$ может достигать $5 \mathrm{BT} / \mathrm{kBT}^{2}$ при накачке на частоте 263 ГГц и величине потерь $\alpha_{2 f}=0.1 \mathrm{~cm}^{-1}$. Показана возможность заметного увеличения эффективности удвоения частоты при тонкой подстройке фазового синхронизма за счет подбора величины субмикрометрового зазора между пластиной InP и обкладками металлического волновода. Полученные результаты могут быть использованы для решения прикладных задач в области спектроскопии динамической поляризации ядер и исследований ядерного магнитного резонанса.
\end{abstract}

Ключевые слова: нелинейные преобразования частоты, терагерцовое излучение, фосфид индия, нелинейность второго порядка.

DOI: $10.21883 /$ FTP.2021.10.51433.31

\section{1. Введение}

В настоящее время ведется активная разработка источников излучения терагерцового (ТГц) диапазона для решения задач как научного, так и прикладного характера [1-4]. Типичными параметрами источника ТГц излучения, который был бы востребован в области спектроскопии, радиоастрономии, мониторинга окружающей среды и вопросах безопасности, являются выходная частота вблизи 1 ТГц и мощность на уровне 1 ВТ в непрерывном режиме при комнатной температуре. В то же время подобные показатели находятся вне пределов досягаемости для источников, основанных на применении генераторов обратной волны [5] и полупроводниковых квантовых каскадных лазеров [6]. Среди прочих подходов молекулярные лазеры ограничены фиксированным набором выходных частот [7], а источники ТГц излучения на основе „оптического выпрямления“ фемтосекундных импульсов (как правило, в кристаллах LiNbO3 [8], $\mathrm{ZnTe}[9], \mathrm{GaP}[10])$ характеризуются широким ( 1 ТГц $)$ спектром излучения, что не всегда приемлемо для приложений спектроскопии, а также довольно низкой выходной мощностью [11]. Наконец, такие источники терагерцового излучения, как синхротроны и лазеры на свободных электронах, чрезвычайно дороги и громоздки, что существенно ограничивает их использование даже в научных задачах.

Альтернативный способ получения интенсивного ТГц излучения - умножение частоты суб-ТГц источников за счет генерации гармоник в нелинейных кристаллах. Источниками излучения накачки здесь могут служить мощные гиротроны суб-ТГц диапазона, которые в настоящее время находятся в стадии значительного прогресса [12,13]. В непрерывном режиме генерации достигнута мощность до 1 кВт на частоте 263 ГГц [14], разработаны непрерывные гиротроны с уровнем мощности в десятки $[15,16]$ и даже сотни [17] Ватт на частотах $\sim 500$ ГГц, в которых достигнута плавная перестройка частоты в полосе $1-2 \%$, импульсные $[18,19]$ и непрерывные [20] гиротроны, работающие на частотах $>1$ ТГц, предложены методы селективного возбуждения высших циклотронных гармоник, что позволяет кратно увеличить частоту генерируемого излучения без увеличения рабочего магнитного поля [21-23]. Для генерации гармоник в полупроводниковых кристаллах зачастую используется электронная нелинейность третьего порядка; так, генерация третьей гармоники гиротронного излучения в $n$-Ge и $\mathrm{Si}$ по такому механизму рассматривалась в [24,25]. Эффективность генерации третьей гармоники (определяемая как отношение мощностей третьей и 
основной гармоник) составила $0.05 \%$ при накачке на частоте 70 ГГ [24] и $0.07 \%$ при накачке на частоте 118 ГГц [25].

Генерация второй гармоники возможна за счет использования решеточной нелинейности второго порядка, присутствующей в кристаллах без центра инверсии. Так, в [26] изучалось удвоение частоты в кристалле GaAs, однако эффективность преобразования оказалась весьма низкой $\left(\sim 10^{-7}\right)$, что связано $\mathrm{c}$ малой величиной нелинейной восприимчивости $\left(\chi^{(2)} \approx 5.7 \cdot 10^{-9}\right.$ см/В) и практическими ограничениями на длину кристалла [27]. Нелинейность второго порядка значительно более выражена в таких материалах, как InP $\left(\chi^{(2)} \approx 3.5 \cdot 10^{-8} \mathrm{~cm} / \mathrm{B}\right), \mathrm{InAs}$ или GaSb [28], однако практические ограничения на выбор нелинейного кристалла определяются также и величиной оптических потерь в кристалле на субтерагерцовых частотах, связанных с решеточным (фононным) поглощением и поглощением на свободных носителях заряда. С этой точки зрения наиболее интересной нелинейной средой является InP:Fe; при современном уровне чистоты материала InP:Fe сочетает в себе высокую нелинейность второго порядка с низкими диэлектрическими потерями в субТГц области спектра $[29,30]$.

Простейшие соображения в соответствии с [31] подсказывают, что наиболее эффективная генерация второй гармоники в нелинейном кристалле может быть реализована при максимальной фокусировке излучения накачки с одновременной реализацией максимальной длины взаимодействия волн (длины когерентности). В данной работе будет рассмотрен один из подходов к решению этой задачи - локализация излучения в пределах возможной более тонкой пластины InP:Fe, помещенной в конический волновод с металлическими обкладками (рис. 1). Ширина пластины $W$ полагается много большей длины волны накачки; в данной работе из практических соображений принято $W=3$ мм. Для определенности мы рассмотрим генерацию второй гармоники при частоте накачки 263 ГГц; выбор данной частоты обусловлен развитием специализованных источников суб-ТГц излучения для спектроскопии динамической поляризации ядер и исследований ядерного магнитного резонанса, работающих на частотах 263, 395, 527 и 593 ГГц (соответствующих частотам электронного парамагнитного резонанса ${ }^{1} \mathrm{H}$ ) с выходной мощностью до 1 кВт. При этом гиротронное излучение характеризуется гауссовым профилем пучка, что позволяет обеспечить эффективный ввод излучения накачки в плоский волновод [32].

Конфигурация волновода, приведенная на рис. 1, позволяет эффективно локализовать электрическое поле волны накачки в нелинейном кристалле, обеспечивая максимальную его интенсивность и соответственно квадратичный выигрыш в интенсивности второй гармоники. Непосредственный контакт пластины InP с одной из стенок волновода может обеспечить эффективный теплоотвод от этой пластины при ее нагреве излучением накачки; даже при относительно небольшой величине

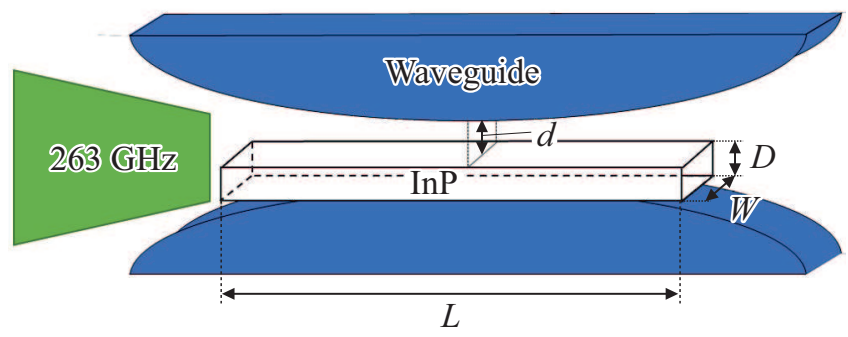

Рис. 1. Схема размещения пластины InP в коническом металлическом волноводе: $D, W$ и $L$ - толщина, ширина и длина пластины соответственно; $d$ - толщина воздушного зазора.

поглощения волны накачки в InP джоулевы потери могут быть заметными при мощности накачки на уровне 1 кВт. Наконец, наличие воздушного зазора между нелинейным кристаллом и второй стенкой волновода позволяет, как будет показано далее, в некоторых пределах „Подстроить“ фазовый синхронизм волн на основной и удвоенной частотах и добиться более эффективного преобразования частоты. В данной работе будет рассмотрено влияние геометрических факторов в системе волновод/нелинейный кристалл на интенсивность излучения второй гармоники при заданной мощности пучка накачки.

\section{2. Расчетная модель}

Развивая подход, приведенный в [33], мы рассмотрим удвоение частоты в кристалле InP, выращенном на плоскости (001), в котором излучение накачки основная гармоника гиротрона, поляризованная в плоскости (001), - распространяется вдоль направления [110]. Такая геометрия практически удобна, поскольку позволяет завести накачку через боковые грани кристалла, сколотые по плоскостям типа (110). В этом случае в системе координат, где ось $x$ направлена вдоль направления распространения волн [110], ось $y-$ вдоль направления $[\overline{1} 10]$ и ось $z-$ вдоль $[001]$, отличной от нуля будет только одна $y$-компонента электрического поля в волне накачки:

$$
E_{y}(x, z, t)=A(z)\left\{\exp \left(i k_{x}, x-i \omega t\right)+\exp \left(-i k_{x}, x+i \omega t\right)\right\} .
$$

Далее, кристалл InP имеет структуру цинковой обманки (симметрия $T_{d}$ ), за счет чего тензор нелинейной диэлектрической восприимчивости второго порядка $\chi_{i j k}^{(2)}$, записанный в координатах вдоль кристаллографических осей $[100],[010]$ и [001], симметричен относительно перестановки индексов. Кроме того, отличными от нуля являются только те его компоненты, в которых все три индекса не равны друг другу; соответственно нелинейную диэлектрическую восприимчивость среды можно описать всего лишь одной функцией частоты: $\chi_{i j k}^{(2)}(\omega)=\chi^{(2)}(\omega)$. При этих условиях вектор нелинейной 
поляризации будет направлен строго перпердикулярно плоскости волновода, вдоль оси $z$ :

$$
P_{z}=-\chi^{(2)} E(x, z, t)^{2} \text {. }
$$

Таким образом, генерация второй гармоники в рассматриваемой системе возможна только в ТМ-поляризации. Исходя из (1) и (2) нелинейную поляризацию среды, ответственную за генерацию удвоенной частоты, можно представить в следующем виде:

$$
\begin{aligned}
P_{z}= & -\chi^{(2)} A(z)^{2}\left\{\exp \left(2 i k_{x} x-2 i \omega t\right)\right. \\
& \left.+\exp \left(-2 i k_{x}, x+2 i \omega t\right)\right\} .
\end{aligned}
$$

Поскольку очевидным требованием для эффективной генерации второй гармоники является обеспечение возможно большей длины взаимодействия электромагнитной волны на удвоенной частоте с волной нелинейной поляризации, мы будем далее рассматривать только случай коллинеарного распространения излучения основной и удвоенной гармоник. Тогда уравнение для $y$-компоненты магнитного поля $H_{y}^{\prime}(x, z, t)$ ТМ-моды на удвоенной частоте, по аналогии с генерацией разностной частоты в той же геометрии [34], имеет вид

$$
\begin{gathered}
\varepsilon(2 \omega, z) \frac{\partial}{\partial z}\left[\frac{1}{\varepsilon(2 \omega, z)} \frac{\partial H_{y}^{\prime}(x, z, t)}{\partial z}\right]+\frac{\partial^{2} H_{y}^{\prime}(x, z, t)}{\partial x^{2}} \\
-\frac{\varepsilon(2 \omega, z)}{c^{2}} \frac{\partial^{2} H_{y}^{\prime}(x, z, t)}{\partial t^{2}}=-\frac{4 \pi}{c} \frac{\partial}{\partial t}\left(\frac{\partial P_{z}}{\partial x}\right),
\end{gathered}
$$

где $\varepsilon(2 \omega, z)$ - профиль диэлектрической проницаемости для удвоенной частоты: $\varepsilon(2 \omega, z)=\varepsilon(2 \omega)$ в кристалле InP (при $0<z<D), \varepsilon(2 \omega, z)=1$ в воздушном зазоре $(D<z<D+d)$ и $\varepsilon(2 \omega, z)=\varepsilon_{m}$ в алюминиевых обкладках $(z<0$ и $z>D+d)$. Очевидно, что решение уравнения (4) можно представить как удвоенную действительную часть решения аналогичного уравнения, в правой части которого имеется только одна „бегушая“ экспонента, соответствующая одному из слагаемых в (3): $H_{y}^{\prime}(x, z, t)=2 \operatorname{Re}\left[H_{y}(x, z, t)\right]$.

Далее мы будем считать, что длина когерентности (взаимодействия) волн $L_{c}$, определяемая выражением

$$
L_{c}=\left[\left|\mathbf{k}_{2}-2 \mathbf{k}_{1}\right|^{2}+(\alpha / 2)^{2}\right]^{-1 / 2}
$$

(здесь $\mathbf{k}_{1}, \mathbf{k}_{2}$ - волновые вектора волн на основной и на удвоенной частоте, $\alpha-$ коэффициент поглощения в InP на частоте $2 \omega)$, значительно превышает характерную длину затухания излучения второй гармоники: $L_{c} \gg \alpha^{-1}$. В этом случае мы можем искать „стационарное“ решение в виде $H_{y}(x, z, t)=H_{y}(z) \exp \left(i q_{x} x-2 i \omega t\right)$, и уравнение для $H_{y}(z)$ примет вид

$$
\begin{aligned}
\frac{d^{2} H_{y}(z)}{d z^{2}}+\left(\varepsilon(2 \omega) \frac{4 \omega^{2}}{c^{2}}\right. & \left.-q_{x}^{2}\right) H_{y}(z) \\
& =-\frac{16 \pi}{c} k_{x} \omega \chi^{(2)} A(z)^{2} .
\end{aligned}
$$

При этом на границе слоев с разными диэлектрическими проницаемостями непрерывны величины $H_{y}(z)$ и $\varepsilon^{-1}(\omega, z) d H_{y} / d z$, а граничные условия для волноводных мод при $z \rightarrow \pm \infty$ задаются как $H_{y}(z) \rightarrow 0$. Для численного решения (5) и нахождения $H_{y}(z)$ требуется предварительно решить следующее уравнение для его „задающей“ части - профиля поля $A(z)$ волны на основной частоте:

$$
\frac{d^{2} A(z)}{d z^{2}}+\left[\frac{\varepsilon(\omega) \omega^{2}}{c^{2}}-k_{x}^{2}\right] A(z)=0 .
$$

Здесь $\varepsilon(\omega)$ - диэлектрическая проницаемость InP на основной частоте; на границах слоев непрерывны величины $A(z)$ и $d A(z) / d z$, а граничные условия при $z \rightarrow \pm \infty$ имеют вид $A(z) \rightarrow 0$. Компонента электрического поля на удвоенной частоте $E_{z}(z)$ и мощность волны на второй гармонике определяются выражениями:

$$
\begin{aligned}
& E_{z}(z)=\frac{1}{\varepsilon(2 \omega)}\left(4 \pi \chi^{(2)} A(z)^{2}-\frac{c q_{x}}{2 \omega} H_{y}(z)\right), \\
& P_{2 \omega}=-\frac{c W}{2 \pi} \int_{0}^{D+d} \operatorname{Re}\left[H_{y}(z) E_{z}^{*}(z)\right] d z .
\end{aligned}
$$

Для последующего анализа удобно в качестве меры эффективности генерации второй гармоники ввести коэффициент преобразования $\beta=P_{2 \omega} / P_{\omega}^{2}$, где $P$ - общая мощность волны накачки.

\section{3. Результаты и обсуждение}

На рис. 2 представлен расчет эффективности $\beta$ генерации второй гармоники для пластины InP, плотно зажатой между стенками металлического волновода $(d=0)$ при частоте излучения накачки $\omega / 2 \pi=263$ ГГц

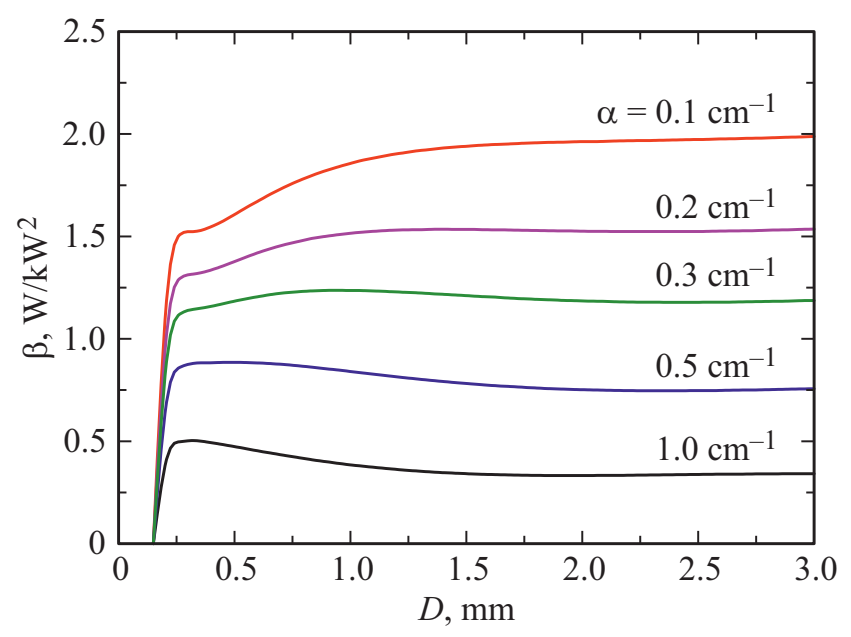

Рис. 2. Зависимость эффективности преобразования $\beta$ от толщины $D$ пластины InP и величины $\alpha$ оптических потерь на второй гармонике (расчет для $d=0$ ). (Цветной вариант рисунка представлен в электронной версии статьи). 


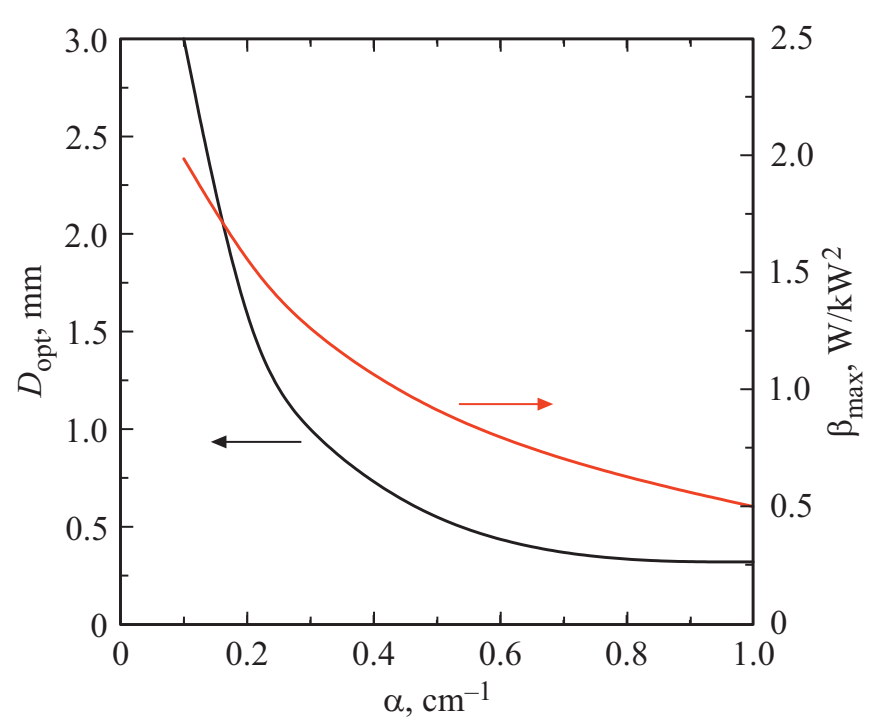

Рис. 3. Зависимость величин $\beta_{\max }$ и $D_{\text {opt }}$ от величины потерь $\alpha$ (расчет для $d=0)$.

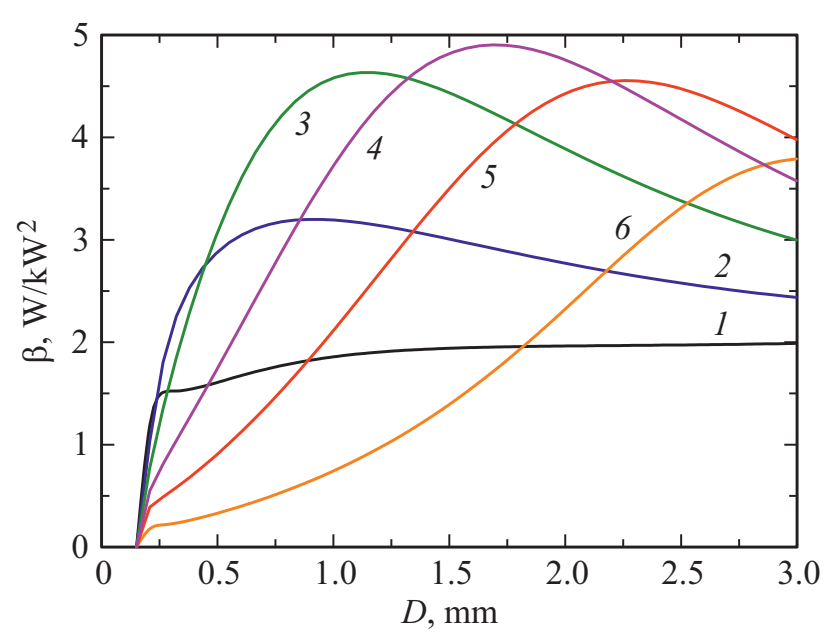

Рис. 4. Зависимость эффективности преобразования $\beta$ от толщины $D$ пластины InP для различных величин воздушного зазора $d$ в волноводе; случай слабых потерь: $\alpha=0.1 \mathrm{~cm}^{-1}$. Расчет для значений $d=0(1), 25(2), 50(3), 75$ (4), 100 (5) и 150 нм (6).

в зависимости от толщины пластины $D$ и величины потерь $\alpha$. Видно, что генерация второй гармоники становится возможной при превышении некой критической толщины пластины InP $D_{\min } \approx 180$ мкм, приблизительно соответствующей критической толщине волновода для основной ТЕ моды на частоте накачки. Далее, увеличение $D$ приводит к уменьшению модовых потерь на удвоенной частоте, однако при фиксированной общей мощности пучка уменьшается также и плотность мощности накачки. Эти противоположно направленные факторы определяют наличие некоторой оптимальной толщины пластины $D_{\text {орt }}$, обеспечивающей максимальный коэффициент преобразования $\beta_{\max }$. Зависимость $D_{\max }$

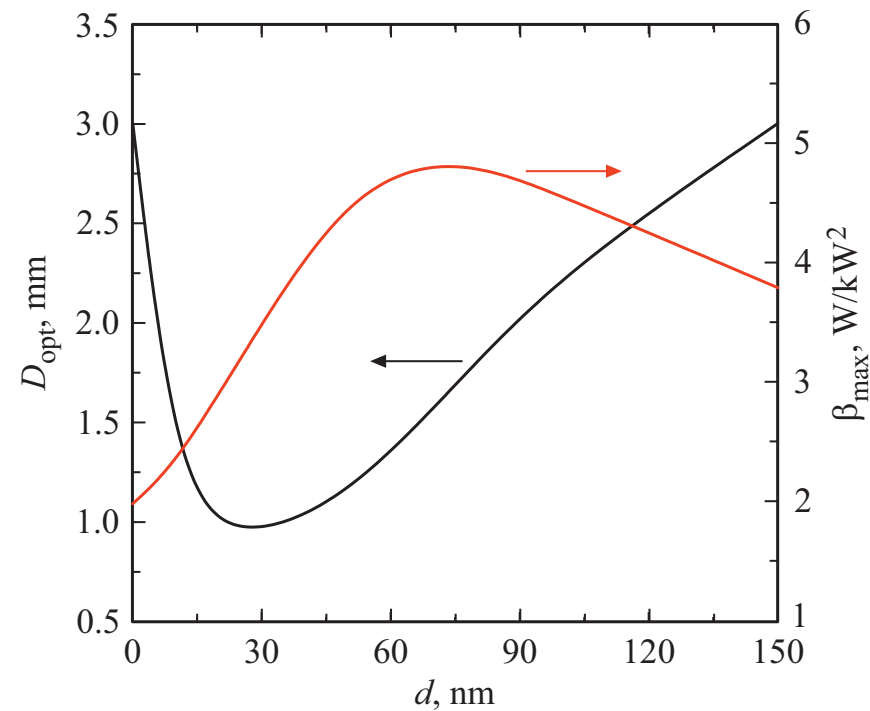

Рис. 5. Зависимость величин $\beta_{\max }$ и $D_{\text {орt }}$ от толщины $d$ воздушного зазора в волноводе; случай слабых потерь: $\alpha=0.1 \mathrm{~cm}^{-1}$.

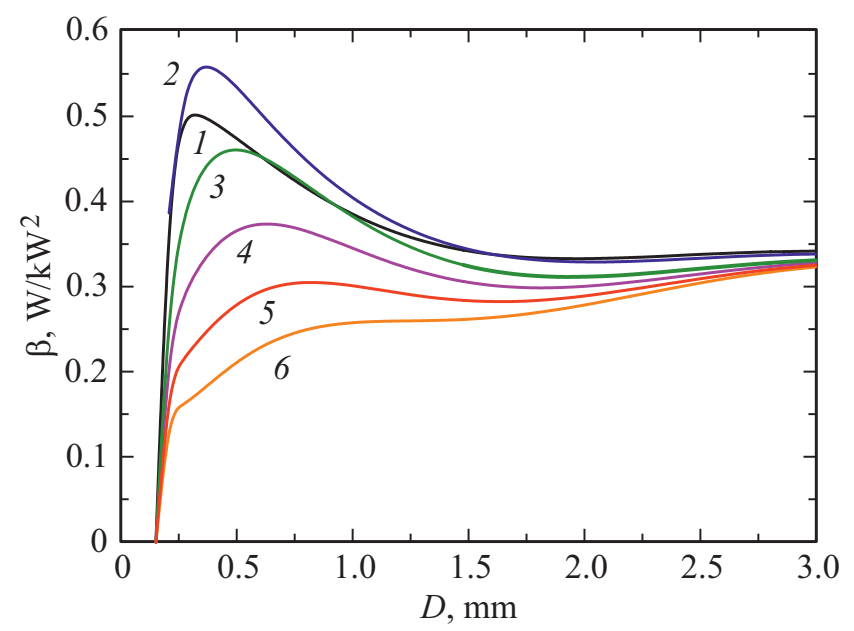

Рис. 6. Зависимость эффективности преобразования $\beta$ от толщины $D$ пластины InP для различных величин воздушного зазора $d$ в волноводе; случай сильных потерь: $\alpha=1 \mathrm{~cm}^{-1}$. Расчет для значений $d=0(1), 25(2), 50(3), 75(4), 100$ (5) и 150 Hм (6).

и $\beta_{\max }$ от величины потерь $\alpha$ приведена на рис. 3 ; с ростом поглощения эффективность преобразования $\beta_{\max }$ уменьшается с 2 до $0.5 \mathrm{BT} / \mathrm{kBT}^{2}$, а оптимальная толщина

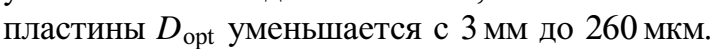

Отметим, что в рассматриваемой системе - пластина InP в металлическом волноводе - в принципе возможна „Подстройка“ фазового синхронизма взаимодействующих волн за счет подбора величины $d$ зазора между InP и стенкой волновода; тем самым можно заметно увеличить эффективность генерации второй гармоники. Соответствующие расчетные данные для случая относительно слабого поглощения на удвоенной частоте $\left(\alpha=0.1 \mathrm{~cm}^{-1}\right)$ приведены на рис. 4 и 5. Видно, что 


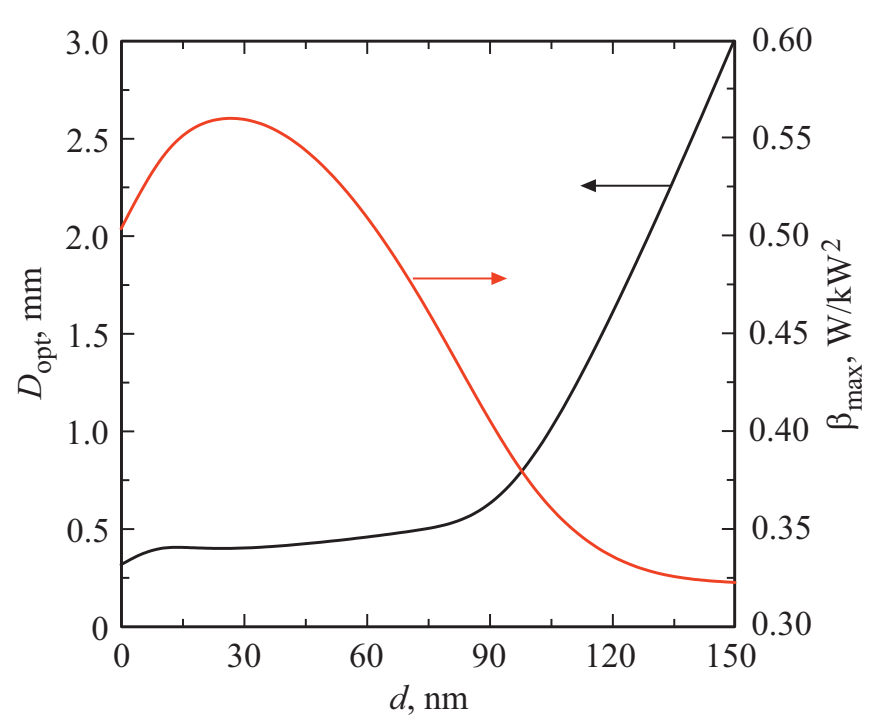

Рис. 7. Зависимость величин $\beta_{\max }$ и $D_{\text {opt }}$ от толщины $d$ воздушного зазора в волноводе; случай сильных потерь: $\alpha=1 \mathrm{~cm}^{-1}$.

при оптимально подобранных величинах $D_{\text {орt }} \approx 1.7$ мм и $d=75$ нм величина $\beta_{\max }$ может достигать $5 \mathrm{BT} / \mathrm{\kappa BT}^{2}$, в 2.5 раза превышая соответствующее значение при отсутствии воздушного зазора $(d=0)$. Для сравнения, на рис. 6 и 7 приведены аналогичные расчетные данные в случае сильного поглощения $\left(\alpha=1 \mathrm{~cm}^{-1}\right)$. Здесь за счет наличия воздушного зазора возможно лишь незначительно повысить эффективность преобразования. Это соответствует интуитивным ожиданиям, поскольку „установившееся“ значение интенсивности волны второй гармоники достигается при меньшей длине взаимодействия волн на основной и удвоенной частотах. За счет этого требования к длине когерентности волн $L_{c}$ становятся менее жесткими, а фазовые поправки, вносимые изменением геометрии, - менее значимыми.

\section{4. Заключение}

В работе проведено моделирование эффективности генерации второй гармоники гиротронного излучения при накачке пластины InP, встроенной в металлический волновод. Показано, что как оптимизация толщины пластины, обеспечивающая локализацию электромагнитного поля волны накачки в нелинейном кристалле, так и подбор величины воздушного зазора между InP и стенками волновода, дающий подстройку фазового синхронизма волн на основной и удвоенной частотах, критически важны для эффективного удвоения частоты в рассматриваемой системе. При оптимальной геометрии и реалистичной величине оптических потерь в кристалле InP (на частоте второй гармоники) $\alpha=0.1 \mathrm{~cm}^{-1}$ возможно получить коэффициент преобразования $\beta \equiv P_{2 \omega} / P_{\omega}^{2}$ на уровне $\sim 5 \mathrm{BT} / \mathrm{kBT}^{2}$; таким образом, при входной мощности 1 кВт ожидаемая эффективность генерация второй гармоники составляет $0.5 \%$.

\section{Финансирование работы}

Работа поддержана грантом Российского научного фонда № 18-79-10112.

\section{Конфликт интересов}

Авторы заявляют, что у них нет конфликта интересов.

\section{Список литературы}

[1] Handbook of terahertz technologies: devices and applications, ed. by Ho-Jin Song, Tadao Nagatsuma (Jenny Stanford Publishing, N. Y., 2015).

[2] J.H. Booske, R.J. Dobbs, C.D. Joye, C.L. Kory, G.R. Neil, G. Park, J. Park, R.J. Temkin. IEEE Trans. Terahertz Sci. Technol., 1 (1), 54 (2011). doi: 10.1109/TTHZ.2011.2151610

[3] R.A. Lewis. J. Phys. D: Appl. Phys., 47 (37), 374001 (2014).

[4] T. Idehara, S. Mitsudo, S. Sabchevski, M. Glyavin, I. Ogawa. Vacuum, 62 (2-3), 123 (2001). https://doi.org/10.1016/S0042-207X(00)00456-5

[5] Xiong Xu, Yanyu Wei, Fei Shen, Hairong Yin, Jin Xu, Yubin Gong, Wenxiang Wang. Phys. Plasmas, 19 (1), 013113 (2012).

[6] B.S. Williams. Nature Photonics, 1 (9), 517 (2007).

[7] S. Ganichev, W. Prettl. Intense Terahertz Excitation of Semiconductors (Oxford University Press, 2005).

[8] S.B. Bodrov, A.A. Murzanev, Y.A. Sergeev, Y.A. Malkov, A.N. Stepanov. Appl. Phys. Lett., 103 (25), 251103 (2013).

[9] S. Vidal, J. Degert, M. Tondusson, E. Freysz, J. Oberlé. J. Opt. Soc. Amer. B, 31 (1), 149 (2014).

[10] R.A. Lewis. J. Phys. D: Appl. Phys., 47 (37), 374001 (2014).

[11] G. Nusinovich, M. Thumm, M. Petelin. J. Infr. Milli Terahz Waves, 35, 325 (2014). https://doi.org/10.1007/s10762-014-0050-7

[12] T. Idehara, S.P. Sabchevski, M. Glyavin, S. Mitsudo. Appl. Sci., 10 (3), 980 (2020). https://doi.org/10.3390/app10030980

[13] M.Yu. Glyavin, M.V. Morozkin, A.I. Tsvetkov, L.V. Lubyako, G.Yu. Golubiatnikov, A.N. Kuftin, V.E. Zapevalov, V.V. Kholoptsev, A.G. Eremeev, A.S. Sedov, V.I. Malygin, A.V. Chirkov, A.P. Fokin, E.V. Sokolov, G.G. Denisov. Radiophys. Quant. Electron., 58, 639 (2016). https://doi.org/10.1007/s11141-016-9636-3

[14] K. Aoki, J. Savolainen, M. Havenith. Appl. Phys. Lett., 110 (20), 201103 (2017).

[15] S.K. Jawla, R.G. Griffin, I.A. Mastovsky, M.A. Shapiro, R.J. Temkin. IEEE Trans. Electron. Dev., 67 (1), 328 (2020).

[16] M. Blank, P. Borchard, S. Cauffman, K. Felch. 43rd Int. Conf. on Infrared, Millimeter, and Terahertz Waves (IRMMWTHz, 2018) p. 1-2. doi: 10.1109/IRMMW-THz.2018.8510010

[17] G.G. Denisov, M.Yu. Glyavin, A.N. Kuftin, M.D. Proyavin, M.V. Morozkin, D.I. Sobolev, A.P. Fokin, E.M. Tai, Yu.V. Rodin, A.G. Luchinin, V.N. Manuilov, A.S. Sedov. 22nd Int. Vacuum Electronics Conf. (2021) p. 1.

[18] M. Glyavin, A. Luchinin. Radiophys. Quantum Electron., 50, 755 (2007). https://doi.org/10.1007/s11141-007-0066-0

[19] I. Bandurkin, V. Manuilov, V. Bratman, I. Osharin, Y. Kalynov, A. Savilov. Int. Vacuum Electronics Conf. (IVEC, 2019) p. 1. doi: 10.1109/IVEC.2019.8745126

[20] T. Idehara, I. Ogawa, H. Mori, S. Kobayashi, S. Mitsudo, T. Saito. J. Plasma Fusion Res. Ser., 8, 1508 (2009). 
[21] I.V. Bandurkin, A.E. Fedotov, A.P. Fokin, M.Yu. Glyavin, A.G. Luchinin, I.V. Osharin, A.V. Savilov. EDL, 41 (9), 1412 (2020). doi: 10.1109/LED.2020.3010445

[22] N.S. Ginzburg, M.Y. Glyavin, A.M. Malkin, V.N. Manuilov, R.M. Rozental, A.S. Sedov, A.S. Sergeev, V.Y. Zaslavsky, I.V. Zotova, T. Idehara. IEEE Trans. Plasma Sci., 44 (8), 1303 (20160. doi: 10.1109/TPS.2016.2585307

[23] M.Y. Glyavin, A.G. Luchinin, V.N. Manuilov, G.S. Nusinovich. IEEE Trans. Plasma Sci., 36 (3), 591. doi: 10.1109/TPS.2008.917530

[24] F. Keilmann, R. Brazis, H. Barkley, W. Kasparek, M. Thumm, V. Erckmann. Europhys. Lett., 11 (4), 337 (1990).

[25] R. Narkowicz, M.R. Siegrist, Ph. Moreau, J.P. Hogge, R. Raguotis, R. Brazis. Acta Phys. Polon. A, 119 (4), 509 (2011).

[26] T. Dekorsy, V.A. Yakovlev, W. Seidel, M. Helm, F. Keilmann. Phys. Rev. Lett., 90 (5), 055508 (2003); A. Mayer, F. Keilmann. Phys. Rev. B, 33 (10), 6954 (1986).

[27] F. Zernike, J.E. Midwinter. Applied Nonlinear Optics (Wiley, N. Y., 1973).

[28] O. Madelung. Semiconductors: Data Handbook (N. Y., Springer-Verlag, 2003).

[29] V.V. Rumyantsev, K.V. Maremyanin, A.P. Fokin, A.A. Dubinov, V.V. Utochkin, M.Yu. Glyavin, N.N. Mikhailov, S.A. Dvoretskii, S.V. Morozov, V.I. Gavrilenko. Semiconductors, 53 (9), 1217 (2019).

[30] L.N. Alyabyeva, E.S. Zhukova, M.A. Belkin, B.P. Gorshunov. Sci. Rep., 7 (1), 7360 (2017).

[31] F. Zernike, J.E. Midwinter. Applied Nonlinear Optics (John Wiley \& Sons Inc., 1973).

[32] M.Yu. Glyavin, A.V. Chirkov, G.G. Denisov, A.P. Fokin, V.V. Kholoptsev, A.N. Kuftin, A.G. Luchinin, G.Yu. Golubyatnikov, V.I. Malygin, M.V. Morozkin, V.N. Manuilov, M.D. Proyavin, A.S. Sedov, E.V. Sokolov, E.M. Tai, A.I. Tsvetkov, V.E. Zapevalov. Rev. Sci. Instr., 86, 054705 (2015). https://doi.org/10.1063/1.4921322

[33] A.A. Dubinov, V.V. Rumyantsev, V.I. Gavrilenko, S.V. Morozov. Optical Engineering, 60 (8), 082014 (2021).

[34] A.A. Dubinov, V.V. Utochkin. J. Appl. Phys., 128, 053104 (2020).

Редактор Л.В. Шаронова

\section{Calculation of the efficiency of doubling the radiation of a sub- $\mathrm{THz}$ gyrotron dueto lattice nonlinearity in a single crystal InP plate}

V.V. Rumyantsev ${ }^{1}$, A.P. Fokin ${ }^{2}$, A.A. Dubinov' ${ }^{1}$, S.S. Morozov' , A.A. Bogdashov', V.V. Parshin', M.Yu. Glyavin ${ }^{2}$, V.I. Gavrilenko ${ }^{1}$, S.V. Morozov ${ }^{1}$

${ }^{1}$ Institute for Physics of Microstructures, Russian Academy of Sciences, 603950 Nizhny Novgorod, Russia

2 Institute of Applied Physics, Russian Academy of Sciences, 603950 Nizhny Novgorod, Russia ${ }^{3}$ Lobachevsky State University, 603950 Nizhny Novgorod, Russia

Abstract In this work, we calculated the efficiency of second harmonic generation for sub- $\mathrm{THz}$ gyrotron radiation in a singlecrystal InP plate embedded in a conical metal waveguide. For values of losses at the doubled frequency (depending on the degree of purity of the InP crystal), the dimensions of the wafer are revealed that provide the optimal effective frequency conversion. Thus, the conversion factor $f \rightarrow 2 f$ can reach $5 \mathrm{~W} / \mathrm{kW}^{2}$ when pumped at the frequency of $263 \mathrm{GHz}$ and the loss of $\alpha_{2 f}=0.1 \mathrm{~cm}^{-1}$. The possibility of a noticeable increase in the efficiency of frequency doubling with fine tuning of the phase matching due to the selection of the size of the submicrometer gap between the InP plate and the plates of the metal waveguide is shown. The results obtained can be used to solve applied problems in the field of dynamic nuclear polarization spectroscopy and nuclear magnetic resonance studies. 